\title{
Data structure for on-lattice cluster-cluster aggregation model performance optimization
}

\author{
Hailing Xiong ${ }^{\mathrm{a}, \mathrm{b}, *}$, Hang $\mathrm{Li}^{\mathrm{c}}$, Weiping Chen ${ }^{\mathrm{d}}$, Laosheng $\mathrm{Wu}^{\mathrm{e}}$ \\ ${ }^{a}$ College of Computer and Information Science, Southwest University, Chongqing 400715, China \\ ${ }^{\mathrm{b}}$ Key Laboratory of Eco-environments in Three Gorges Reservoir Region (Ministry of Education), Southwest University, Chongqing 400715, China \\ ${ }^{\mathrm{c}}$ College of Resources and Environment, Southwest University, Chongqing 400715, China \\ d State Key Lab of Urban and Regional Ecology, Chinese Academy of Sciences, Beijing 100085, China \\ e Department of Environmental Sciences, University of California, Riverside, CA 92521, USA
}

\section{A R T I C L E I N F O}

\section{Article history:}

Received 4 August 2013

Received in revised form

10 November 2013

Accepted 19 November 2013

Available online 25 November 2013

\section{Keywords:}

Data structure

On-lattice simulation

Metropolis sampling

Cluster-cluster aggregation

Computational complexity

\begin{abstract}
A B S T R A C T
A compounded data structure is developed to optimize the simulation of colloidal aggregation using the on-lattice Cluster-Cluster Aggregation (CCA) model. Brownian motion, collision detection and aggregation as the basic operations in the CCA simulation are illustrated and evaluated based on the compounded data structure, respectively. The critical improvement of our algorithm is in distinguishing any selected clusters consisting particles and ascertaining their neighboring positions efficiently in simulation, which was traditionally performed by the exhaustive search in the whole system. Analytical results show that the new algorithm achieves linear computational complexity in each of the main operations, which is very appealing in performance optimization in using on-lattice CCA simulations.
\end{abstract}

(C) 2013 Elsevier B.V. All rights reserved.

\section{Introduction}

Colloidal aggregation is an important environmental phenomenon as pollutants often are present as colloids in water and aerosols in the atmosphere [1-3]. The process starts with a suspension of finely divided particulates at a low initial volume fraction in which stable colloids must overcome the repulsive energy barrier between two particles to coalesce and grow in size [3]. The Cluster-Cluster Aggregation (CCA) model assumes that initially a collection of equal sized individual spherical particles are randomly dispersed in a box at low concentrations and then these particles are allowed to self-diffuse in Brownian motion by random walk, collide, and form clusters [4,5]. The CCA model is developed to simulate the particle growing mechanism [6-9]. It is widely adopted to describe the aggregation of colloids and aerosols with good agreements with experimental results [10-14]. The simulated outcomes provide insights in understanding the aggregation process and determine important features such as fractal dimension and structure

\footnotetext{
* Corresponding author at: College of Computer and Information Science, Southwest University, Chongqing 400715, China. Tel.: +86 2368367731 ; fax: +86 2368252051.

E-mail address: xionghl@swu.edu.cn (H. Xiong).
}

factor of clusters, shapes of cluster mass distribution, and aggregation kinetics [12].

The CCA models perform well in small-scale simply simulations. The computation processes however challenge the capabilities of current day digital computers requiring large memories and high speed processors. The computational complexity is a significant handicap to simulate colloidal systems comparable with real-world experimental data $[15,16]$. Hawick, etc. had publicized a serious of technical reports about the simulation method, one technical report similar to this research discussed space partitioning methods for the CCA simulation and demonstrate complexity improvements by taking advantage of information about locations and interaction distances of the microscopic model components [16]. Kusaka, etc. studied CCA simulation in a concentrated suspension, reported on the characteristics of the collision radius of fractal aggregates, and discussed aggregation kinetics based on the value of the estimated collision radius [13]. The CCA model is authentically a recognized and widely used ingenious model to mimic fractal aggregation. However, emphasis has long been focused more on the structure of the aggregates and the kinetics of aggregation processes than on the algorithm efficiency.

The conventional algorithm of a CCA model is depicted in Fig. 1 [17] in which the Brownian movement, collision detection and aggregation are the main steps. The simulation starts with $N$ non-overlapping identical particles distributed randomly in a cubic 


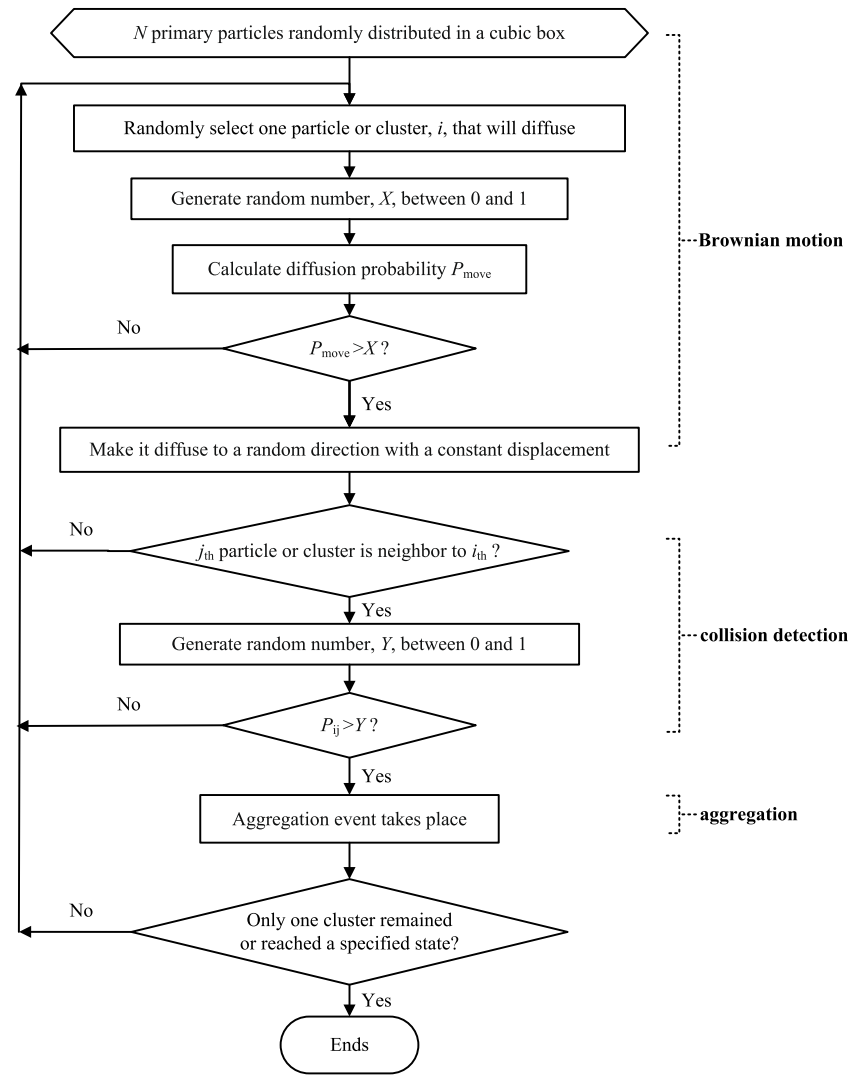

Fig. 1. Algorithmic flow chart of on-lattice CCA simulations.

box with side-lengths of $L$. In Brownian movement, all particles are in motion and all particles have chance to collide in realistic situations. For the CCA models, Meakin separates and serializes the Brownian movements by adopting the Metropolis sampling method [4]. It lies on the basic premise that in simulations only one randomly selected particle (or cluster) moves at a time and with the distance identical to diameter of the primary particle [6].

To realize the CCA simulation, a three dimensional array, $C U B E[L][L][L]$, was used to represent the cubic box. Each particle in the cubic box occupies an element of the three dimensional array and are labeled with a different integer $(>0)$, therefore on-lattice. When particles and/or clusters collide and aggregate, all particles in the resulting cluster have the same label Fig. 2 . The progression of Brownian movement and aggregation are realized by updating the labels of the corresponding array elements.

A critical issue of the simulation is how to efficiently distinguish all of the particles in any selected cluster based on the three dimensional array CUBE $[L][L][L]$ when the cluster is to be moved. Similarly, there are difficulties in the process of collision detection to locate all neighboring positions of the cluster. For example, if a cluster labeled with $i$ was selected to undergo Brownian movement i.e. self-diffuse, all elements that are identified by the label $i$ in the three dimensional array $C U B E[L][L][L]$ must be tested. It is a time-consuming process of $\mathrm{O}\left(L^{3}\right)$ computational complexity in three dimensional simulations because an exhaustive search of the entire system must be performed upon each simulated Brownian motion. According to the flow chart (Fig. 1) only the selected cluster need to be checked in the collision detection step. It nevertheless remains to be a time-consuming process because the clusters are quite anomalous and the labels of particles in the selected cluster have to be checked for collision detection one by one with those in all other clusters. A three dimensional array CUBE $[L][L][L]$ is not efficient for the on-lattice CCA simulations.

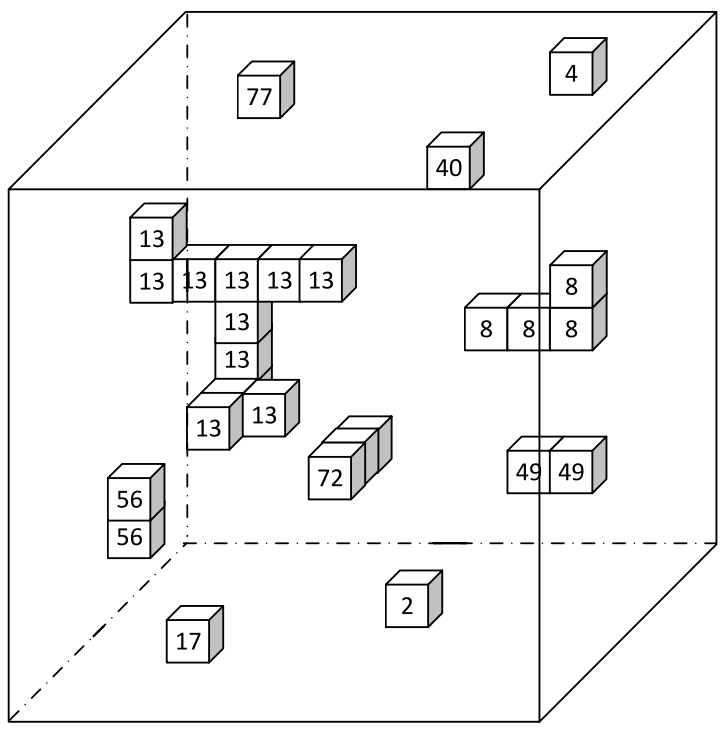

Fig. 2. Single particles and clusters in the cube (in part) are distinguished by the labels marked on the little boxes, which are stored in the corresponding array elements.

We have developed a compounded data structure for the onlattice CCA simulation that improves the performance efficiency of the simulation algorithm. In the following sections we delineate the simulation model, new data structure, algorithm realization, and efficiency evaluation.

\section{Simulation model}

The details of the CCA model are given as follows [6]: we take the unit $a=1$ for a lattice constant. In the model, $N$ particles are randomly distributed in a cubic box with side-length of $L$. Thus, the particle concentration $c$ becomes:

$c=N / L^{3}$

Assuming the shape effects can be ignored, the diffusion coefficient $D_{i}$ of a cluster (consists of $i$ particles) is proportional to the inverse of its gyration radius $R_{g i}$ [18]:

$D_{i}=D_{1} \times R_{1} / R_{g i}$

where $D_{1}$ is the diffusion coefficient of the single particle with the gyration radius of $R_{1}$.

According to the Metropolis sampling method, one cluster will be selected in each step for a potential movement. The selection is accepted or rejected based on the probability $P_{\text {move }}[4]$ :

$P_{\text {move }}=D_{i} / D_{\max }$

where $D_{i}$ is the diffusion coefficient of the selected cluster, $D_{\max }$ is the maximum diffusion coefficient for any cluster in the system. For the potential movement, a random number $x$ uniformly distributed over the range $[0,1]$ is generated and the cluster is moved only if $x<P_{\text {move }}$.

The moving direction is also randomly chosen among six directions. If the cluster does not collide with another one, the displacement is performed and the algorithm goes on by choosing another cluster for the next step. If a collision occurs between two clusters (one consists of $i$ particles, and another consists of $j$ particles) they stick together forming a new larger cluster with the sticking probability $P_{i j}[4,12]$ :

$P_{i j}=P_{1} \times(i \times j)^{\sigma}$ 


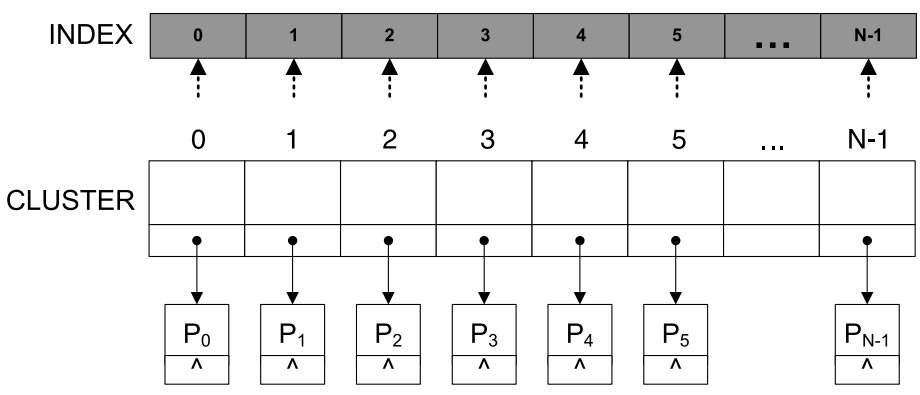

(a)

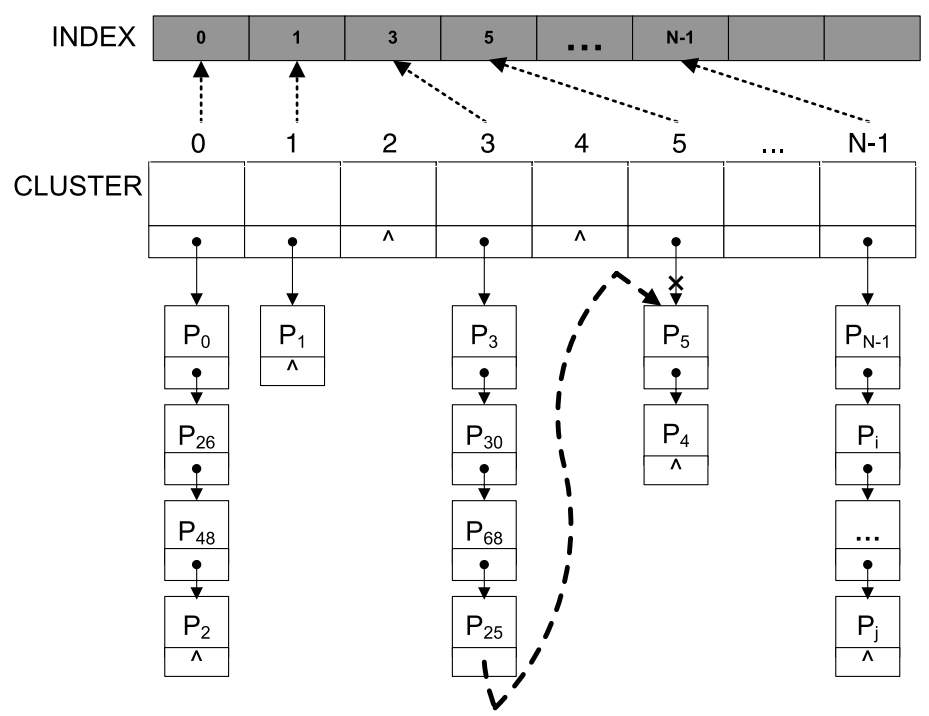

(b)

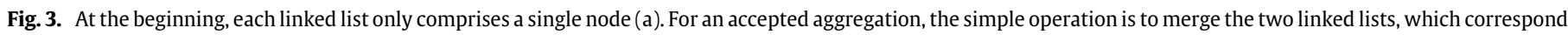

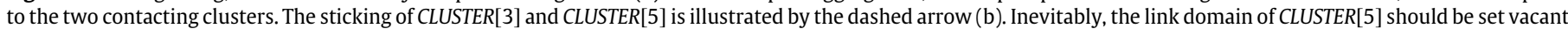

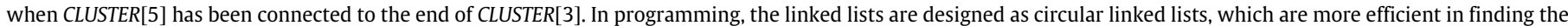
last node for the connection.

where $P_{1}$ is the sticking probability of single particles, $\sigma$ is the sticking probability exponent, by which two distinct extreme regimes, i.e., the Diffusion-Limited Cluster-Cluster Aggregation (DLCA) and the Reaction-Limited Cluster-Cluster Aggregation (RLCA) can be distinguished. In DLCA, $P_{1}=1$ and $\sigma=0$, while in RLCA, $P_{1}<1$ and $\sigma \neq 0 . P_{i j}$ is set to unity if $P_{1}(i j)^{\sigma}>1[4,19,20]$. Simultaneously, a random number $y$ uniformly distributed over the range of 0 to 1 is generated and compared with the calculated $P_{i j}$. The sticking is considered effective only when $y<P_{i j}$ is verified [18].

In on-lattice simulation, a large cube is divided into equal-size little boxes [14]. For easy management of computation, the three dimensional large cube has equal size in each dimension. The sidelength of the little box is regarded as a unit length. In this manner, the conventional Cartesian coordinates of each box can be easily specified.

\section{Data structure}

We also use a three dimensional array $C U B E[L][L][L]$ represents the system space, where $L$ is equal to the side length of the cube. Each cluster in the system is assigned a label, such as a positive integer number. The corresponding elements in the array store the corresponding number to differ with each other. At initial time, each single particle is treated as a cluster with a unique label since they are placed without contact. The elements in the array with the values of -1 (to be different from those particle or cluster labels in the system) mean where the dispersion medium is. When a particle or cluster moves, the element it previously occupied reverts back to -1 if it was vacant at the time.

Differing from the previous simple three dimensional array approaches, we use additional linked lists to store the information of clusters in the system, respectively, in which each linked list node corresponds to a single particle of the cluster. The particle position information is stored in the node, by which we can easily search the particle in the array CUBE $[L][L][L]$. In this manner, the traditional exhaustive search in the whole system is totally avoided because the array elements can be directly accessed.

Another one dimensional array CLUSTER $[N]$ is used to organize the linked lists and store the basic attributes of the clusters in the system. Initially, $N$ linked lists, each only has one node, are created for the $N$ randomly placed particles (Fig. 3a). After a cluster is moved, its perimeter is examined to determine whether any other clusters have been contacted via nearest neighbor occupancy. If aggregation does occur $\left(y<P_{i j}\right)$ after the collision detection, the simple operation is to link the corresponding clusters (Fig. 3b).

After an aggregation, the array CLUSTER should be indexed simultaneously because there may be some vacant linked lists, such as CLUSTER[2] and CLUSTER [4] in Fig. 3b. In the later random selection for the potential movement, these vacant linked lists should 
not be in the candidate lists. For the indexing, a one dimensional array INDEX $[N]$ is used to store the non-vacant linked list subscripts of the array CLUSTER[N]. The current length of the INDEX is the number of the remaining clusters in the system. For example, if the new generated random number for the random selection is $k$, the label of the next moving cluster should be INDEX $[k]$, and the linked list of this cluster is exactly linked by CLUSTER [INDEX $[k]]$. From this entry, all the particles in the cluster can be easily accessed. And then, the positions of the six neighboring boxes of each particle in the cluster can also be accessed by the element subscripts of the array CUBE, which is needed for the collision detection.

In summary, the programming for Brownian motion, collision detection and aggregation of clusters becomes very efficient when the new data structure is adopted, which can be further verified in the next section.

\section{Algorithm realization and efficiency evaluation}

It is difficult to compare our new method with the previous methods because the details of those methods were almost not introduced completely. Therefore, we analyze and evaluate the main steps in our on-lattice CCA simulation with the classical algorithm analysis method.

\subsection{Brownian motion}

The parallel Brownian motions of clusters are serialized in computer simulations by using the Metropolis sampling method as mentioned above. In this way, only the selected cluster would be moved a unit length in one of the six directions at one time step. There are many clusters in the system, and each cluster is assigned a unique label, which is stored in the three dimensional array. Every element in the array with a positive integer number corresponds to a real particle. All the elements with the same positive integer number are regarded as belonging to the same cluster. With the help of the array CLUSTER, it is very easy to find the entry of the linked list (the moving cluster) by using CLUSTER[INDEX [k]] since the array CLUSTER has been indexed after every combination. The coordinates of all the particles are stored in the linked list, thus, one simply needs to process the corresponding elements in the array CUBE one by one, instead of going through the whole three dimensional array CUBE. This means that only one of the coordinates $x, y$ or $z$ will be modified uniformly by adding or subtracting one unit for the operation of Brownian movement. Based on the new data structure, the time complexity of the Brownian motion algorithm can be reduced to $\mathrm{O}(N)$. It is obviously very appealing in time-saving since the large number of Brownian motion in simulation is one of the fundamental operations.

\subsection{Collision detection}

Following the Brownian motion, collision detection would be performed immediately. There are six neighboring boxes of a single particle. If the core is a particle with the coordinate $(x, y, z)$, the neighbors are the boxes sharing a face with the core, which are specified with the coordinates $(x+1, y, z),(x-1, y, z),(x, y+$ $1, z),(x, y-1, z),(x, y, z+1)$ and $(x, y, z-1)$. By the coordinates we can directly access the values stored in the three dimensional array CUBE. If one of them has a different positive value with the core, the aggregation detection based on the $P_{i j}$ will be processed immediately. As mentioned above, we already have the entry of the linked list (the moving cluster) by using CLUSTER[INDEX[k]], the nodes in the linked list can be directly accessed, by which the neighboring boxes of the particles in the cluster also can be accessed directly. It is evident that the compounded data structure is efficient in saving simulation time since its time complexity is only at the order of $\mathrm{O}(N)$ for the collision detection, which is far less than that in an $\mathrm{O}\left(N^{2}\right)$ algorithm [16].

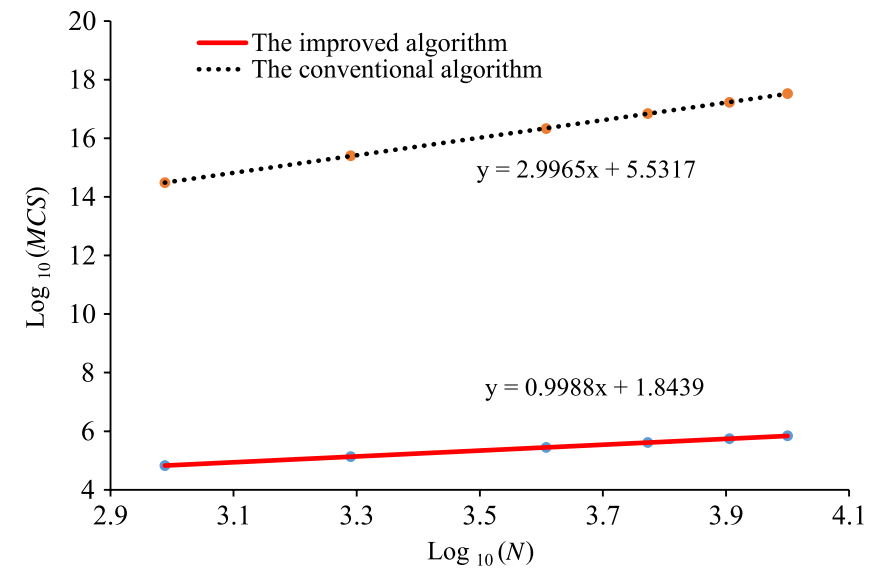

Fig. 4. Log-log plot of simulation time (Monte Carlo steps: $M C S)$ vs. number of particles $(N)$. For constant concentration $(c=0.01), N$ varies in $1000,2000,4000$, 6000,8000 and 10000 .

\subsection{Aggregation}

Another important operation in the simulation process is the aggregation of two contacting clusters. Based on the new data structure, we just need to merge the corresponding linked lists directly as shown in Fig. 3. After a combination of two clusters, it generally needs to calculate the gyration radius, diffusion coefficient and other attributes of the new formed cluster. This process is commonly referred to as system update. Most of the attributes are related to the cluster morphology, which is consequently determined by the specific positions of all the particles in the cluster. Therefore, the feasibility of easily finding out the particles in the huge system is also a great contribution for the system update. In our simulation, this aim is achieved by using the compounded data structure mentioned above.

\subsection{Computational complexity}

A Log-log plot of simulation time vs. number of particles for constant concentration ( $c=0.01$ in this simulation) is shown in Fig. 4. According to the Eq. (1) mentioned above, the number of particles $N$ varies with the side length $L$ of the cube. For constant concentration we just need to adjust the side length $L$ to get different particle numbers. In order to avoiding the hardware and software influence, our simulation time was described as usual by the Monte Carlo steps(MCS) [21,22].

We can see from the fitted equation of the improved algorithm that the coefficient of $x$ is 0.9988 , which is very close to 1 . It means that the computational complexity of the improved algorithm is linear, i.e. $\mathrm{O}(N)$, while that of the conventional algorithm is $\mathrm{O}\left(N^{3}\right)$. Obviously, the result is also corresponding with the previous analysis.

\section{Summaries}

A compounded data structure for on-lattice CCA model is presented. We can easily distinguish the particles belonging to any selected cluster without exhaustive searching the whole system attributed to the direct location of the linked lists in array CLUSTER. Consequently, their neighbors for collision detection can also be directly accessed. This is a great contribution to the operations of Brownian motion, collision detection, aggregation and system update. The new compounded structure only requires an order $\mathrm{O}(N)$ computational complexity in each of the main operations in the on-lattice CCA simulation. 


\section{Acknowledgments}

The authors would like to thank Professor Andrew C. Chang at the University of California, Riverside, USA for his useful advice. This work was financially supported by the National Natural Science Foundation of China (41271292), the Natural Science Foundation Project of CQ (CSTC-2011BB7004), the Doctoral Foundation Project of the Southwest University, China (No. SWUB2008073) and the Fundamental Research Funds for the Central Universities, China (No. XDJK2010C032).

\section{References}

[1] N. Loh, C. Hampton, A. Martin, D. Starodub, R. Sierra, A. Barty, A. Aquila, J. Schulz, L. Lomb, J. Steinbrener, Nature 486 (2012) 513.

[2] L. Matthews, V. Land, T. Hyde, Astrophys. J. 744 (2012) 8.

[3] G. Munaò, Z. Preisler, T. Vissers, F. Smallenburg, F. Sciortino, Soft Matter 9 (2013) 2652.

[4] P. Meakin, J. Sol-Gel Sci. Technol. 15 (1999) 97.

[5] H. Xiong, Y. Yuan, H. Li, H. Zhu, X. Jiang, Acta Phys.-Chim. Sin. 23 (2007) 1241.
[6] R. Jullien, R. Botet, Aggregation and fractal aggregates, World Scientific Singapore, 1987

[7] R. Klein, P. Meakin, Nature 339 (1989) 360

[8] O. Vormoor, Comput. Phys. Commun. 144 (2002) 121.

[9] S. González, A. Thornton, S. Luding Comput. Phys. Commun. 182 (2011) 1842

[10] M. Lin, H. Lindsay, D. Weitz, R. Ball, R. Klein, P. Meakin, Phys. Rev. A 41 (1990) 2005.

[11] M. Lin, H. Lindsay, D. Weitz, R. Klein, R. Ball, P. Meakin, J. Phys.: Condens. Matter 2 (1990) 3093

[12] P. Sandkühler, M. Lattuada, H. Wu, J. Sefcik, M. Morbidelli, Adv. Colloid Interface Sci. 113 (2005) 65.

[13] Y. Kusaka, T. Fukasawa, Y. Adachi, J. Colloid Interface Sci. 363 (2011) 34.

[14] C. Qian, H. Li, R. Zhong, M. Luo, G. Ye, Chinese Physics B 18 (2009) 1947.

[15] K.A. Hawick, Proc. International Conference on Modeling, Simulation and Visualization Methods (MSV10). Number CSTN-012, Las Vegas, USA, MSV3277, 2010.

[16] K.A. Hawick, H.A. James, C. Scogings, Proc. 39th Simulation Symposium, Huntsville, Alabama, USA, April 2006.

[17] S. Kim, K.S. Lee, M.R. Zachariah, D. Lee, J. Colloid Interface Sci. 344 (2010) 353.

[18] H. Xiong, H. Li, W. Chen, J. Xu, L. Wu, J. Colloid Interface Sci. 344 (2010) 37.

[19] Z. Tan, X. Zou, W. Zhang, Z. Jin, Phys. Rev. E 62 (2000) 734.

[20] S. Kempf, S. Pfalzner, Comput. Phys. Commun. 137 (2001) 225

[21] T. Vicsek, F. Family, Phys. Rev. Lett. 52 (1984) 1669.

[22] P.A. Netz, D. Samios, Macromol. Theory Simul. 3 (1994) 607. 